\title{
Análise transversal do ensino da implantodontia no curso de graduação
}

\author{
Cleverton Corrêa Rabelo*, Thomaz Wassall**, Jose Gustavo Sproesser*** \\ * Aluno do Mestrado em Implantodontia, CPO São Leopoldo Mandic \\ ** Coordenador do Mestrado em Implantodontia, CPO São \\ Leopoldo Mandic \\ *** Professor Titular, Mestrado em Implantodontia, CPO São \\ Leopoldo Mandic
}

\section{RESUMO}

O objetivo deste trabalho foi avaliar o ensino da Implantodontia na graduação nas faculdades brasileiras. Realizamos estudo transversal baseado em questionários enviados aos coordenadores de todas as faculdades brasileiras regularmente inscritas no Conselho Federal de Odontologia, ${ }^{5}$ para avaliar o conhecimento da ciência Implantodontia disponibilizado aos alunos de graduação. Constatamos que a disciplina Implantodontia está presente em 56,96\% das faculdades de Odontologia pesquisadas e, nas faculdades onde a disciplina inexiste, os conhecimentos sobre a Implantodontia são ministrados, na maioria das vezes, na disciplina de cirurgia buco-maxilo-facial, o que poderia trazer ao aluno uma visão fragmentada predominantemente cirúrgica da Implantodontia; a carga horária de conhecimentos teóricos e práticos sobre Implantodontia são expressivamente maiores nas faculdades onde a disciplina existe, comparada às faculdades onde os conhecimentos são ministrados como parte de outras disciplinas; não houve comprovação da associação entre o fato da disciplina não ser ofertada e carga horária total do curso; os professores responsáveis pelo ensino da Implantodontia, em mais de dois terços das faculdades não possuem especialidade em Implantodontia, podendo ser a falta de corpo docente qualificado o motivo da ausência da disciplina em quase metade das escolas brasileiras. Diante dos resultados, sugerimos a incorporação da disciplina Implantodontia na grade curricular promovendo melhor embasamento científico para os futuros profissionais.

\section{DESCRITORES}

Implantodontia. Osseointegração. Ensino. Currículo. Graduação.
$\Delta$ reabilitação dentária por meio de implantes osseointegráveis tem se mostrado como um método seguro dentre as alternativas terapêuticas na reabilitação bucal.

Apesar dos altos índices de sucesso, os insucessos ainda são motivos de preocupação; dentre as causas principais de insucesso, o planejamento inadequado ou a imperícia técnica merecem especial atenção por representar um problema de estruturação na formação dos profissionais que se dedicam à Implantodontia.

Maior controle sobre a formação dos implantodontistas poderia ser alcançado se a Implantodontia como disciplina fosse incluída na grade curricular dos cursos de graduação. A exemplo de outras especialidades, conhecimentos básicos e fundamentais poderiam ser administrados desde a graduação. ${ }^{8}$

Chappell $^{4}$ (1974) realizou pesquisa-questionário avaliando o ensino da Implantodontia em 61 escolas norte-americanas, canadenses e porto-riquenhas. Trinta e uma escolas (50.8\%) realizavam cirurgias para instalação de implantes. Os alunos realizavam cirurgias para instalação de implantes em duas escolas enquanto uma escola respondeu que os alunos assistiam as cirurgias e oito escolas responderam que os alunos restauravam os implantes proteticamente. Segundo o autor, 30 escolas (50\%) afirmaram a realização de pesquisas na área de Implantodontia.

Bertolami² (2001) ressalta que o currículo odontológico deve corresponder às evidências atuais: alterações demográficas das doenças atuais e projeções futuras, avanços em ciência e tecnologia e uma transformação sócio-cultural da população afetando a demanda de pacientes para tratamento e capacidade de pagar por ele.

Donoff $^{7}$ (2001) relatou que se a decadente necessidade de reabilitações com próteses totais removíveis 
transfere esse tratamento para uma determinada especialidade e devemos estar prontos para transferir mais conhecimentos sobre Implantodontia no currículo de graduação.

No Brasil, o aumento da expectativa de vida foi comprovado pelos dados do IBGE ${ }^{9}$ (2002), entre 1992 e 2001, a população de mais de 60 anos passou de $7,9 \%$ para $9,1 \%$ da população brasileira, trazendo acréscimo na faixa populacional mais afetada pelo edentulismo.

Huebner $^{8}$ (2002), avaliou grupos de alunos que receberam ou não treinamento laboratorial em Implantodontia durante a graduação. Os alunos da CUSD (Creighton University School of Dentistry), cujo programa propicia aos alunos conhecimentos e prática clínica de Implantodontia, apresentaram melhores resultados comparados aos alunos da MWDS (Midwestern Dental School), onde não é ofertado laboratório e clínica em Implantodontia. Como prática clínica, os graduados pela CUSD exercem a Implantodontia em maiores proporções. Estatisticamente, os graduados da CUSD também referenciam mais pacientes aos especialistas para realização de implantes.

Petropoulos et al..$^{11}$ (2006), relataram pesquisa realizada em 2004 entre os representantes de 56 faculdades de Odontologia dos Estados Unidos e Canadá em uma conferência da ADEA (Associação Americana de Ensino Odontológico). A disciplina de Implantodontia estava presente em $97 \%$ das escolas.

Addy et al. ${ }^{1}(2008)$ pesquisaram as escolas de Odontologia no Reino Unido e Irlanda com respeito ao ensino da Implantodontia na graduação. Das 15 escolas, $13(87 \%$ ) proviam treinamento em Implantodontia e 4 escolas (27\%) possibilitavam aos alunos executarem tratamento com implantes em pacientes. Sete escolas $(46 \%)$ ofereciam aos alunos o estudo de plano de tratamento com implantes e possibilitavam aos alunos a observação e confecção de prótese sobre implantes. Em 5 escolas (33\%) os alunos observavam cirurgia de instalação de implantes. Apenas 1 escola permitia a instalação de implantes pelos alunos. Os departamentos responsáveis pela Implantodontia foram conjuntamente Odontologia restauradora e cirurgia buco-maxilo-facial em 8 escolas, e somente a Odontologia restauradora em 5 escolas.

De Bruyn et $a l_{.}{ }^{6}$ (2009) avaliou a situação do ensino da Implantodontia na graduação através de formadores de opinião de 18 países europeus presentes no Workshop da Associação Européia de Educação Odontológica. A carga horária média da disciplina é de 36 horas; em $57 \%$ das faculdades é permitido aos alunos assistir e em $50 \%$ executar procedimentos restauradores; assistem ou auxiliam cirurgias em 53\% e apenas 5\% executam procedimentos cirúrgicos de instalação de implantes. Pós-graduações na área de Implantodontia são ofertadas em $90 \%$ das faculdades. As barreiras para inserção da disciplina foram a falta de tempo no currículo, falta de corpo docente especializado e falta de subsídios financeiros.

Sanz \& Saphira ${ }^{12}$ (2009) relataram que, a Implantodontia na graduação traz uma tendência para se focar no apropriado treinamento médico para os cirurgiões-dentistas e aumentar a competência no manejo com pacientes com problemas sistêmicos.

McAndrew et al. ${ }^{10}(2010)$, reportaram consenso do encontro da British Society of Prosthetic Dentistry's Education Group, em 2009, com respeito à integração da Implantodontia no currículo de graduação. $\mathrm{O}$ treinamento adequado dos graduados é fundamental para a inserção do uso de próteses implanto-suportadas como corrente principal para o tratamento bem sucedido de pacientes edêntulos.

Por se tratar de uma ciência complexa, que exige destreza e habilidades múltiplas para uma prática segura e eficaz, e também devido à ausência de um padrão de nivelamento da formação dos alunos que ingressam nos cursos de habilitação em Implantodontia, realizamos uma avaliação do ensino da Implantodontia durante a graduação nas faculdades brasileiras durante o ano de 2008.

\section{MATERIAL E MÉTODOS}

A técnica metodológica proposta foi baseado segundo estudo transversal através de pesquisa de campo. Foi utilizado um questionário com perguntas objetivas e mistas, dicotômicas e de múltipla escolha. O questionário é composto por perguntas quantitativas (carga horária do curso, duração do curso, alunos por classe, carga horária total do conteúdo, carga horária de laboratório, carga horária das clinicas) e qualitativas (demais perguntas). Em relação ao tipo de observação, foram utilizadas variáveis independentes em relação à variável analisada (cidade do curso, existência de pós-graduação), mas a quase totalidade das perguntas foram compostas por variáveis dependentes, fatores que diretamente se relacionam ao desempenho da disciplina junto ao curso.

Os questionários foram enviados aos coordenadores de todos os cursos de Odontologia no Brasil, reconhecidos pelo Conselho Federal de Odontologia com formação regular, totalizando 188 faculdades. 


\section{RESULTADOS}

Os resultados desta pesquisa refletem as respostas apresentadas por 79 faculdades de Odontologia do país, através de seus coordenadores com representação de todas as regiões brasileiras e suas dependências administrativas, em um universo de 188 faculdades, o que representa $42 \%$ de taxa de retorno.

Das 79 faculdades brasileiras respondentes, $88,60 \%$ ensinam algo relacionado ao tema Implantodontia e pouco mais da metade tem a disciplina como parte integrante do currículo.

A análise estatística foi realizada pela análise descritiva não paramétrica de homogeneidade das proporções utilizando o teste do qui-quadrado e correlação de Pearson, estabelecendo-se o nível de significância de $5 \%(\mathrm{p}<0,05)$. As variáveis quantitativas foram pareadas e analisadas a variação de sua correlação visando estudar o grau de interdependência dos seus resultados.

Com respeito ao desempenho no ENADE3 do ano 2007, das faculdades de Odontologia, apesar do desempenho estatísticamente superior das escolas públicas frente às particulares ( $\mathrm{p} \leq 0,05)$, não observamos diferenças significativas quando comparamos as faculdades que oferecem ou não a disciplina de Implantodontia na graduação $(\mathrm{p} \geq 0,05)$ (Tabela 1$)$.

Com respeito às aulas de laboratório, a carga horária média entre as escolas que possuem a disciplina

Tabela 1 - Nota no ENADE das faculdades por natureza, com ou sem a disciplina Implantodontia.

\begin{tabular}{|c|c|c|}
\hline Faculdades & Com a disciplina & Sem a disciplina \\
\hline Privadas & $2,82 \mathrm{Ba}( \pm 0,74)$ & $2,86 \mathrm{Ba}( \pm 0,73)$ \\
\hline Públicas & $4,10 \mathrm{Aa}( \pm 0,57)$ & $4,00 \mathrm{Aa}( \pm 1,00)$ \\
\hline
\end{tabular}

Fonte: Dados dos questionários. foi quase cinco vezes superior às que não possuem a disciplina, e a carga horária média de clínica foi quase três vezes superior.

Aulas de laboratório de Implantodontia estão presentes em 39,20\% das escolas, 50,6\% das escolas realizam cirurgias demonstrativas para instalação de implantes e 40,51\% fazem demonstrações clínicas de restaurações protéticas sobre implantes. Os alunos fazem as cirurgias em $5,06 \%$ das escolas e fazem as próteses em $7,59 \%$ das faculdades.

No que tange as atividades clínicas, mais de dois terços das escolas que possuem a disciplina, realizam cirurgias demonstrativas $(68,90 \%)$ contra menos de um terço $(26,50 \%)$ das escolas sem a disciplina. Mais da metade das escolas com a disciplina $(55,60 \%)$ permitem aos alunos acompanharem a reabilitação protética enquanto pouco mais de um quinto das escolas sem a disciplina $(20,60 \%)$ os permitem. Onde o tema faz parte do currículo, $60 \%$ das escolas ofertam aulas de laboratório, enquanto somente $11,80 \%$ das escolas que não tem a disciplina oferecem prática de laboratório (Gráfico 1).

Com respeito à carga horária total do curso, cargas horárias médias muito próximas foram encontradas entre as faculdades que possuem ou não possuem a disciplina.

Porém, é significativamente superior a carga horária destinada ao ensino da Implantodontia nas faculdades brasileiras que possuem a disciplina (54,02 horas), representando mais que o dobro em comparação com as que não possuem a disciplina $(23,47$ horas), conseqüentemente maior quantidade de informações a serem passadas aos alunos.

Com respeito à especialidade do professor responsável pelo ensino da Implantodontia, encontramos dados significativamente diferentes entre as escolas. Entre as faculdades que possuem a discipli-

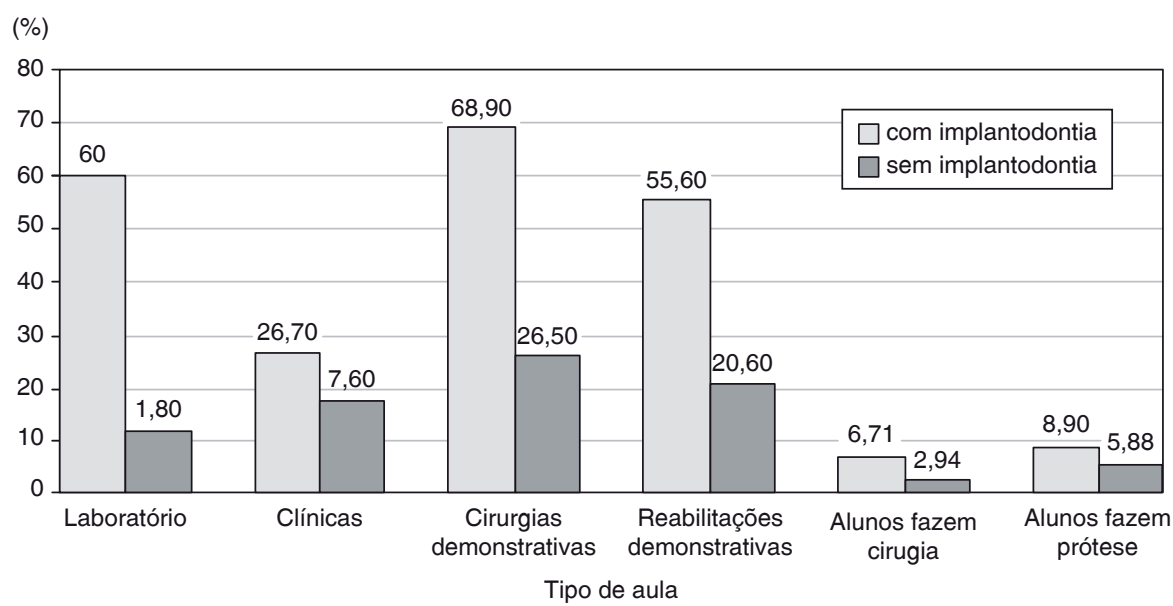

Gráfico 1 - Atividades clínicas em faculdades com ou sem a disciplina Implantodontia. 


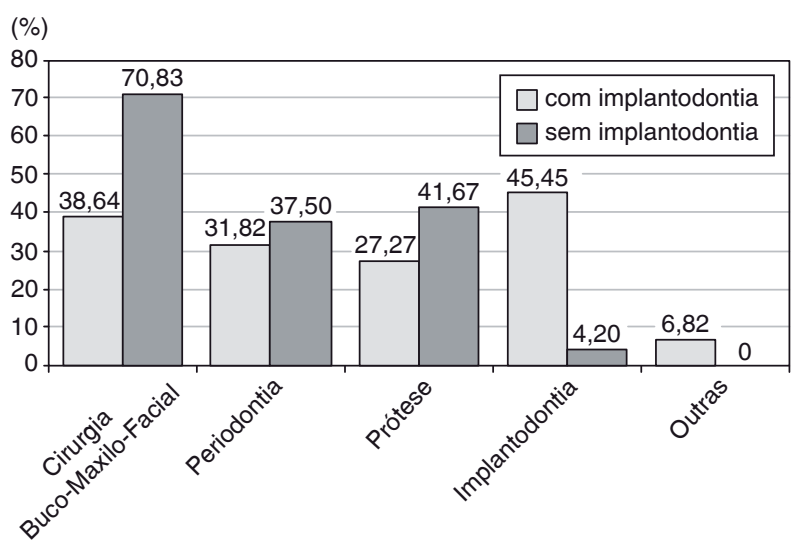

Gráfico 2 - Especialidade do professor responsável.

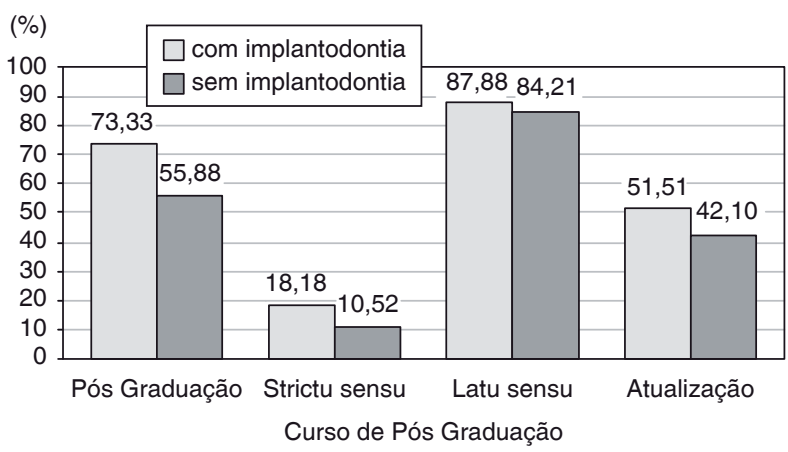

Gráfico 4 - Modalidades de Pós-graduações em Implantodontia nas faculdades com ou sem a disciplina.

na, em $45 \%$ das escolas, o professor responsável tem especialidade em Implantodontia, este percentual cai para $4,17 \%$ nas faculdades que não ofertam a disciplina. Nas faculdades sem a disciplina a maioria dos professores tem a especialização em cirurgia buco-maxilo-facial $(70,83 \%)$ enquanto nas faculdades onde existe a disciplina, $38,64 \%$ tem a mesma especialização. É importante ressaltar que foi possível responder mais de uma especialidade para cada professor (Gráfico 2).

Nas faculdades brasileiras onde a disciplina Implantodontia não existe, os conhecimentos relacionados à Implantodontia são apresentados principalmente na disciplina de cirurgia buco-maxilo-facial (56\%) enquanto $24 \%$ são abordados na disciplina de prótese, e $20 \%$ nas áreas de periodontia e clínica integrada (Gráfico 3).

Observamos que a presença da disciplina na graduação parece estar relacionada à maior oferta de educação continuada. No grupo que possui a disciplina, quase um quinto das escolas que possuem pósgraduações oferecem a modalidade sricto sensu (Gráfico 4).

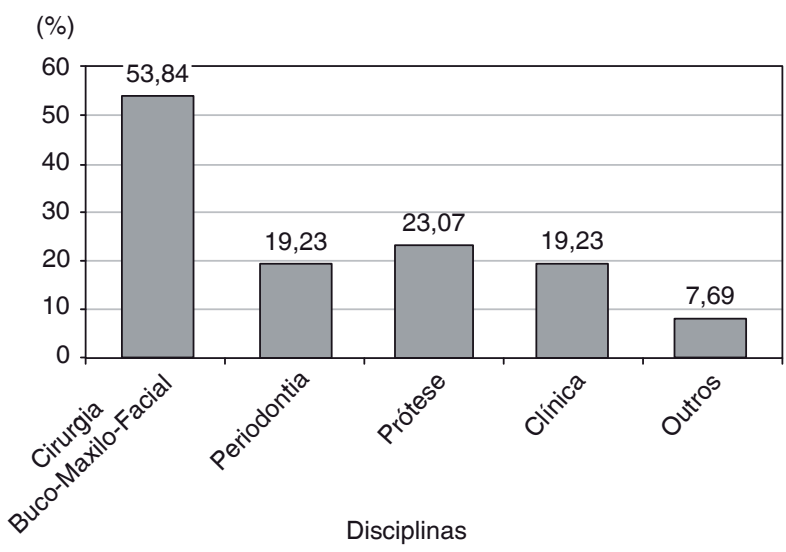

Gráfico 3 - Disciplinas que ofertam o tema nas faculdades que não tem a disciplina Implantodontia.

Com relação aos recursos pedagógicos utilizados, apresentação de casos clínicos são referenciados nas respostas dos coordenadores com frequência quase três vezes maior $(60 \%)$ nas faculdades que oferecem a disciplina em comparação com as que não tem a disciplina $(23,53 \%)$.

Mais da metade das faculdades $(51,16 \%)$ oferecem os conhecimentos sobre Implantodontia, no oitavo período do curso, quando os alunos, geralmente tem maior conhecimento clínico sobre as terapias odontológicas.

\section{DISCUSSÃO}

Segundo pesquisas, o número de desdentados totais está diminuindo, mas o número total de pessoas edêntulas não é menor. Há mais adultos com mais dentes presentes e seus problemas dentais se tornam mais complexos e difíceis de tratar, e isto implica, numa necessidade de mudança na formação dos estudantes de Odontologia, frente aos novos paradigmas que se apresentam. ${ }^{1}$

O resultado deste estudo poderá situar os coordenadores dos cursos de Odontologia sobre o tema em foco, dado a importância do assunto que oferece. No Brasil, a disciplina Implantodontia ainda hoje não preenche os currículos de quase a metade das escolas brasileiras.

Das 79 faculdades brasileiras respondentes, $88,60 \%$ ensinam algo relacionado ao tema Implantodontia e pouco mais da metade $(56,96 \%)$, tem a disciplina como parte integrante do currículo.

$\mathrm{Na}$ revista de literatura, os motivos de algumas escolas não ofertarem a disciplina foram relacionados à falta de recursos financeiros, falta de tempo dentro da grade curricular, ênfase nos programas de pós- 
graduações na área de Implantodontia e insuficiente corpo docente especializado. ${ }^{1,11}$

Quando questionamos sobre a especialidade do professor responsável pelo ensino da Implantodontia, encontramos dados significativamente diferentes entre as escolas. Entre as faculdades que possuem a disciplina, em $45 \%$ das escolas, o professor responsável tem especialidade em Implantodontia, este percentual cai para $4,17 \%$ nas faculdades que não ofertam a disciplina; podendo então discutir se não seria a falta de corpo docente qualificado o motivo para a ausência da disciplina no currículo. ${ }^{11}$

Nas faculdades brasileiras onde a disciplina Implantodontia não existe, os conhecimentos relacionados à Implantodontia são apresentados na maioria das vezes, na disciplina de cirurgia buco-maxilo-facial, contrastando com metodologias internacionais, ${ }^{1,4}$ onde existe abordagem multidisciplinar entre os departamentos envolvidos na terapia com implantes. $\mathrm{O}$ predomínio do departamento de cirurgia buco-maxilo-facial entre as escolas que não têm a disciplina Implantodontia corrobora com o trabalho de Huebner $^{8}$ (2002) onde os recém-formados oriundos de escolas que não tinham a disciplina Implantodontia estruturada na graduação referenciavam seus pacientes para especialistas em cirurgia buco-maxilo-facial 10 vezes mais que outra especialidade, denotando uma visão fragmentada predominantemente cirúrgica da Implantodontia.

Em nossa pesquisa, a qualificação das escolas frente ao desempenho no $\mathrm{ENADE}^{3}$ e sua dependência administrativa não foram significativos com relação ao fato da disciplina ser ofertada. A Implantodontia faz parte do conteúdo avaliativo do $\mathrm{ENADE}^{3}$ e seria de se esperar melhor desempenho dos cursos que possuem a disciplina. É importante a qualificação do corpo docente responsável além de destinar carga horária adequada ao conteúdo de forma que possibilite ao aluno um aprendizado substancial, com correlação prática e teórica.

A presença da disciplina na graduação teve uma correlação positiva com a oferta de pós-graduações na mesma área, não configurando em nosso estudo um motivo para a ausência da disciplina. Pelo contrário, os cursos de educação continuada podem possibilitar oportunidades de pesquisas, estudos triados, e diversos intercâmbios com a graduação.

A carga horária total dos cursos apontada como saturada e justificativa para ausência da Implantodontia, não foi significativamente diferente entre os dois grupos de nossa pesquisa. As médias de cargas horá- rias foram semelhantes, independentemente da presença da disciplina.

Porém, é significativamente superior a carga horária destinada ao ensino da Implantodontia nas faculdades brasileiras que possuem a disciplina, conseqüentemente maior tempo em sala de aula, favorece maior quantidade de informações a serem passadas aos alunos.

Com respeito às aulas de laboratório, a carga horária média entre as escolas que possuem a disciplina foi quase cinco vezes superior às que não possuem a disciplina, e a carga horária média de clínica foi quase três vezes superior. A importância de atividades clínicas e laboratoriais no programa de ensino de Implantodontia foi relacionada a melhor desempenho em prática, melhor capacidade de planejamento e maior interesse em educação continuada. ${ }^{8}$

No que tange as atividades clínicas, mais de dois terços das escolas que possuem a disciplina, realizam cirurgias demonstrativas contra menos de um terço das escolas sem a disciplina. Mais da metade das escolas com a disciplina permitem aos alunos acompanharem a reabilitação protética enquanto pouco mais de um quinto das escolas sem a disciplina os permitem.

Com relação aos recursos pedagógicos utilizados, a apresentação de casos clínicos foi referenciada com freqüência quase três vezes maior nas escolas que tem a disciplina, reforçando a importância de estudos direcionados de associação teórico-demonstrativo, possíveis devido às atividades práticas.

A relevância deste tema persiste na importância desta ciência para os profissionais da Odontologia de uma forma geral e o dever das instituições de oferecer ao aluno a oportunidade de receber conhecimentos nesta área, favorecendo conseqüentemente a comunidade que pode ser muito beneficiada por profissionais melhores formados.

\section{CONCLUSÃO}

- A Implantodontia, como disciplina está presente em 56,96\% das faculdades de Odontologia pesquisadas.

- Nas faculdades onde a disciplina inexiste, os conhecimentos sobre a Implantodontia são ministrados, na maioria das vezes $(53,84 \%)$, na disciplina de Cirurgia Buco-Maxilo-Facial.

- A carga horária de conhecimentos teóricos e práticos sobre Implantodontia são expressivamente maiores nas faculdades onde a disciplina existe, favorecendo o aprendizado e experiência clínica. 
- Não houve comprovação da associação entre o fato da disciplina não ser ofertada e a carga horária total do curso.

- Não houve correlação entre o fato da disciplina não ser ofertada na graduação com o ensino de pós-graduação.

- Os professores responsáveis pelo ensino da Implantodontia, em mais de dois terços das faculdades não possuem especialidade em Implantodontia.

\section{ABSTRACT}

\section{Cross-sectional study of implant dentistry teaching} in the undergraduate course

The objective of this study was to evaluate the teaching of implant dentistry in colleges in Brazil. We conducted a cross-sectional study based on questionnaires sent to the coordinators of all the Brazilian colleges regularly enrolled in the Federal Council of Dentistry, ${ }^{5}$ to assess the level of knowledge of the science of implant dentistry available to undergraduate students. We found that the academic subject of implant dentistry was found in $56.96 \%$ of the dental schools and colleges surveyed. In those colleges where the discipline does not exist, implant dentistry is mostly taught in the course on maxillofacial surgery. This approach could give the student a fragmented, predominantly surgical view of implant dentistry. The workload of theoretical and practical dental implant subject matter is significantly higher in schools where the discipline is offered, compared to the colleges where the subject matter is taught as part of other disciplines. There was no evidence of an association between the fact that the course is not offered and the total hours of the course. The teachers responsible for teaching implant dentistry in more than two thirds of Brazilian colleges have no expertise in this field. The reason why this discipline does not exist in almost half of Brazilian schools may be because of a lack of a qualified teaching staff. In view of these results, we suggest that the subject of implant dentistry be included in the curriculum of dental schools to establish a better scientific basis for future professionals.

\section{DESCRIPTORS}

Implantology. Osseointegration. Dental education. Undergraduate. •

\section{REFERÊNCIAS}

1. Addy, L.D. et al.. The teaching of implant dentistry in undergraduate dental schools in the United Kingdom and Ireland. British Dental Journal, 2008 Dec.; 205(11): 609-614.

2. Bertolami, C.N. Rationalizing the dental curriculum in light of current disease prevalence and patient demand for treatment: form vs. content. Journal of Dental Education, 2001 Aug.;65 (8):725-735.

3. Brasil. Ministério da Educação. Provão 2007. Sistema de avaliação da educação superior. INEP. Ministério da Educação. Relatório-Síntese, 182p. 2008.

4. Chappell, R.P. Dental school survey. Oral Implantol., 1974; $5(1): 24-32$.

5. Conselho Federal de Odontologia [homepage na internet]. Quadro estatístico das faculdades de odontologia existentes no Brasil no ano de 2008 [acesso em 10 out 2008]. Disponível em: http://www.cfo.org.br/wp-content/uploads/2008/09/ quadro_estatistico_faculdade.pdf

6. De Bruyn, H. et al.. A survey on undergraduate implant dentistry education in Europe. Eur J Dent Educ, 2009; 13 (Supplement 1): 3-9.

7. Donoff, R.B. Commentary on Bertolami Article. Journal of Dental Education, 2001 Aug.;65(8):739-741.

8. Huebner, G.R. Evaluation of a Predoctoral Implant Curriculum: Does Such a Program Influence Graduates' Practice Patterns? Int. J. Oral Maxillofac Implants, 2002;17(4): 543-549.

9. IBGE. Censo Demografico - 2002. Instituto Brasileiro de Geografia e Estatistica [Site Oficial]Disponível em: http://www. ibge.gov.br/home/estatistica/populaçao/perfilidoso/default.shtm

10. McAndrew, R. et al.. Embedding implants in undergraduate dental education. British Dental Journal, 2010 Jan; 208(1):910.

11. Petropoulos, V.C. et al.. Teaching implant dentistry in the predoctoral curriculum: a report from the ADEA implant workshop's survey of deans. Journal of Dental Education, 2006 May.;70(5): 580-588.

12. Sanz, M.; Saphira, L. Competencies in implant therapy for the dental graduate. Appropriate educational methods. EurJ Dent Educ, 2009; 13(Supplement 1): 36-43. 